\title{
EL ÍNDICE NAO Y LA PRECIPITACIÓN MENSUAL EN LA ESPAÑA PENINSULAR
}

\author{
Javier Martín Vide \\ David Fernández Belmonte \\ Departamento de Geografía Física y Análisis Geográfico Regional \\ Universidad de Barcelona
}

\section{RESUMEN}

En el artículo se realiza un análisis detallado a resolución mensual de la correlación existente entre un índice NAO y la precipitación en la España peninsular. El índice NAO se ha construido a partir de la presión atmosférica en superficie en Lisboa y Reykjavik, mientras que las series de precipitación mensual utilizadas corresponden a 41 observatorios durante el período 1900-1994. Los resultados obtenidos permiten distinguir cuatro grupos de meses: de octubre a marzo, inclusives, con buena correlación negativa entre el índice NAO y la precipitación en el centro y suroeste de la Península Ibérica, lo que permite regionalizar el área de estudio en tres regiones; abril y mayo, con una dependencia pluviométrica apreciable, aunque menor que en los meses anteriores; el trimestre estival, de junio a agosto, sin señal NAO; y el mes de septiembre, que puede considerarse de transición entre el trimestre estival y el período de clara significación de la señal NAO.

Palabras clave: correlación, España peninsular, índice NAO, precipitación mensual.

\begin{abstract}
A detailed analysis in a monthly basis of the correlation between a NAO index and the precipitation for peninsular Spain is made. The NAO index is calculated by means of Lisbon and Reykjavik sea surface pressure series. The monthly series of precipitation of 41 Spanish observatories for the period 1900-1994 are used. The results allow to distinguish four groups of months: (1) From October to March, with high negative correlation between the NAO index and the precipitation in the centre and the Southwest of peninsular Spain -this permits to regionalize the country into three regions; (2) April and May with less dependence of precipitation from NAO index; (3) the summer (June, July and August) without NAO signal; and (4) September, a transitional month between the summer and the months with clear NAO signal.
\end{abstract}

Kew words: correlation, monthly precipitation, NAO index, peninsular Spain. 


\section{Introducción}

Es bien conocido que la Oscilación del Atlántico Norte (NAO, North Atlantic Oscillation) tiene una influencia destacada en las anomalías meteorológicas y en la variabilidad climática de la Europa occidental al norte del paralelo 45 (Van Loon and Rogers, 1978; Meehl and Van Loon, 1979; Parker and Folland, 1988; WMO, 1996, etc.). El índice NAO, al relacionar los valores de presión en un punto de las islas Azores, u otro lugar próximo del Atlántico subtropical, y uno de Islandia, o punto cercano del Atlántico subpolar, evalúa con claridad la intensidad y zonalidad de los flujos atlánticos sobre la Europa templada, entre las zonas subpolar y subtropical. La Península Ibérica queda, en cambio, en gran medida, bajo la dinámica de los anticiclones subtropicales, al menos durante la mitad cálida del año. En la mitad fresca o fría, los flujos de poniente sí que afectan a todo el espacio ibérico, aunque a menudo alcanzan la vertiente mediterránea oriental muy modificados. A todo ello se une, en el caso de la España peninsular, por una parte, una orografía compleja, compartimentada y con una gran altitud media $(600 \mathrm{~m})$, que actúa de obstáculo para la circulación de los flujos atlánticos hacia el este. Y, por otra, la dinámica atmosférica propia de la cuenca del Mediterráneo, especialmente activa en otoño y principios de invierno, por tener sus aguas una notable anomalía térmica positiva, produce situaciones atmosféricas en las tierras españolas más próximas al Mediterráneo poco comparables a las del centro y el norte de Europa. No ha de olvidarse, tampoco, que la franja más septentrional del país no es mediterránea, bajo ningún concepto, sino que tiene un claro carácter oceánico templado. Todo ello convierte a la España peninsular, con sus casi medio millón de km², en un espacio de una gran complejidad climática y, específicamente, pluviométrica (Gil Olcina y Morales Gil, 1989; Martín Vide, 1996; Martín Vide y Gómez, 1999; Capel Molina, 2000), con una sensibilidad variada ante la Oscilación del Atlántico Norte.

En el presente trabajo se hallan las correlaciones entre un índice NAO y la precipitación de 41 observatorios españoles peninsulares para cada uno de los meses del año. El procedimiento de las correlaciones NAO-precipitación, para intervalos temporales estacionales o mensuales, ha sido ampliamente usado (Katz, 1988). La cartografía del coeficiente de correlación revela comportamientos muy diferenciados según las regiones, que detallan los resultados a menor resolución espacial ya conocidos (Zorita et al., 1992; Hurrell, 1995; Rodó et al., 1997) y permite, con otros índices, establecer una regionalización pluviométrica del territorio. Se amplían, asimismo, temporalmente los resultados obtenidos en un estudio anterior para el mes de diciembre (Martín Vide et al., 1999). Se precisan, así, los resultados generales que asocian los períodos secos en España a elevados (positivos) índices NAO y se avanza en el conocimiento de las teleconexiones en el Mediterráneo occidental (Laita, 1995).

\section{Metodología del trabajo}

Con el objeto de que el índice NAO sea lo más explicativo posible de la compleja pluviometría española, se ha elegido como observatorio meridional uno ibérico, entre los más occidentales de la Península: Lisboa (38.43 N; $9.08 \mathrm{~W}$ ), ya utilizado en otros casos (Hurrell, 1995). La presión en superficie en Lisboa está íntimamente ligada a la potencia y la proximidad del anticiclón de las Azores, que con frecuencia proyecta una dorsal anticiclónica hacia las tierras ibéricas. Como observatorio septentrional se ha elegido Reykjavik $(64.09 \mathrm{~N} ; 21.51 \mathrm{~W})$, que, junto a Lisboa, forma parte de la base de datos barométricos de la Climatic Research Unit (University of East Anglia), utilizada aquí. 
Los 41 observatorios pluviométricos son los mejores de primer orden disponibles para la España peninsular (Ministerio de Medio Ambiente, 1996). Con el objeto de utilizar el mayor número de estaciones de total garantía se ha elegido el periodo 1900-1994. Anteriormente a 1900 funcionaron un cierto número de ellas, aunque con registros más incompletos.

El índice NAO utilizado es la diferencia entre los valores estandarizados de la presión media mensual en Lisboa y en Reykjavik. Para ello, las dos series barométricas de partida se convierten en series de valores $Z$, restándolas de las correspondiente medias y dividiendo estas diferencias por la desviación tipo. La diferencia entre las dos series de valores $Z$ (Lisboa-Reykjavik) da lugar a la serie de índices NAO.

Una vez calculados los índices NAO para cada mes se han ensayado las correlaciones, mediante la $r$ de Pearson, con las precipitaciones mensuales correspondientes de cada observatorio. Los valores obtenidos iguales o superiores a 0.2 , en valor absoluto, serán en todos los observatorios, excepto los que presenten lagunas, significativos según la F de Fisher-Snedecor con $\alpha=0.05$.

La hipótesis general a comprobar es la de una correlación negativa entre el índice NAO y la precipitación, es decir, valores positivos han de comportar períodos secos y valores negativos, episodios lluviosos.

\section{Incidencia del índice NAO sobre la precipitación mensual en la España peninsular}

La influencia general del índice NAO en la precipitación de la Península Ibérica puede dividirse en tres períodos. Un primer período es el compuesto por los meses de octubre, noviembre, diciembre, enero, febrero y marzo. En este período las correlaciones generales del índice NAO y la precipitación son elevadas en valor absoluto y negativas, con las diferencias regionales que se señalarán. Un segundo período lo compone los meses de abril y mayo, en los cuales las correlaciones todavía son significativas, aunque sensiblemente menores. El tercer período está formado por el trimestre estival, junio, julio y agosto, durante el cual la incidencia del índice NAO queda difusa o es inexistente. Queda, finalmente, septiembre, que constituye un mes de transición entre el verano y el periodo de máxima dependencia entre la NAO y la precipitación, por lo que se tratará de forma individualizada.

\subsection{Enero}

En el mes de enero la presión media en Lisboa es de 1022.1 hPa y en Reykjavik, 998.2 $\mathrm{hPa}$, lo que supone una variación de casi $24 \mathrm{hPa}$. Las desviaciones típicas son de $4.3 \mathrm{hPa}$ para Lisboa y $9.9 \mathrm{hPa}$ para Reykjavik, lo que refleja la conocida mayor variabilidad bárica de las latitudes subpolares respecto a las subtropicales. El valor más alto del índice NAO corresponde a 1986, con 3.56, mientras que el valor menor se encuentra en 1963, con - 4.79.

En este mes todos los valores de la $r$ de Pearson obtenidos para la correlación entre el índice NAO y la precipitación son negativos, encontrándose, como valor más bajo, -0.67 en San Fernando, mientras que el más alto es -0.07 en Bilbao. Las correlaciones son significativas en todos los observatorios utilizados para el estudio, excepto en Bilbao, San Sebastián y Murcia, donde los valores no alcanzan 0.2 en valor absoluto. En la parte meridional y central de la Península es donde se hallan los valores de correlación más altos de la $r$, entre $-0.5 \mathrm{y}-0.6$, claramente significativos. 


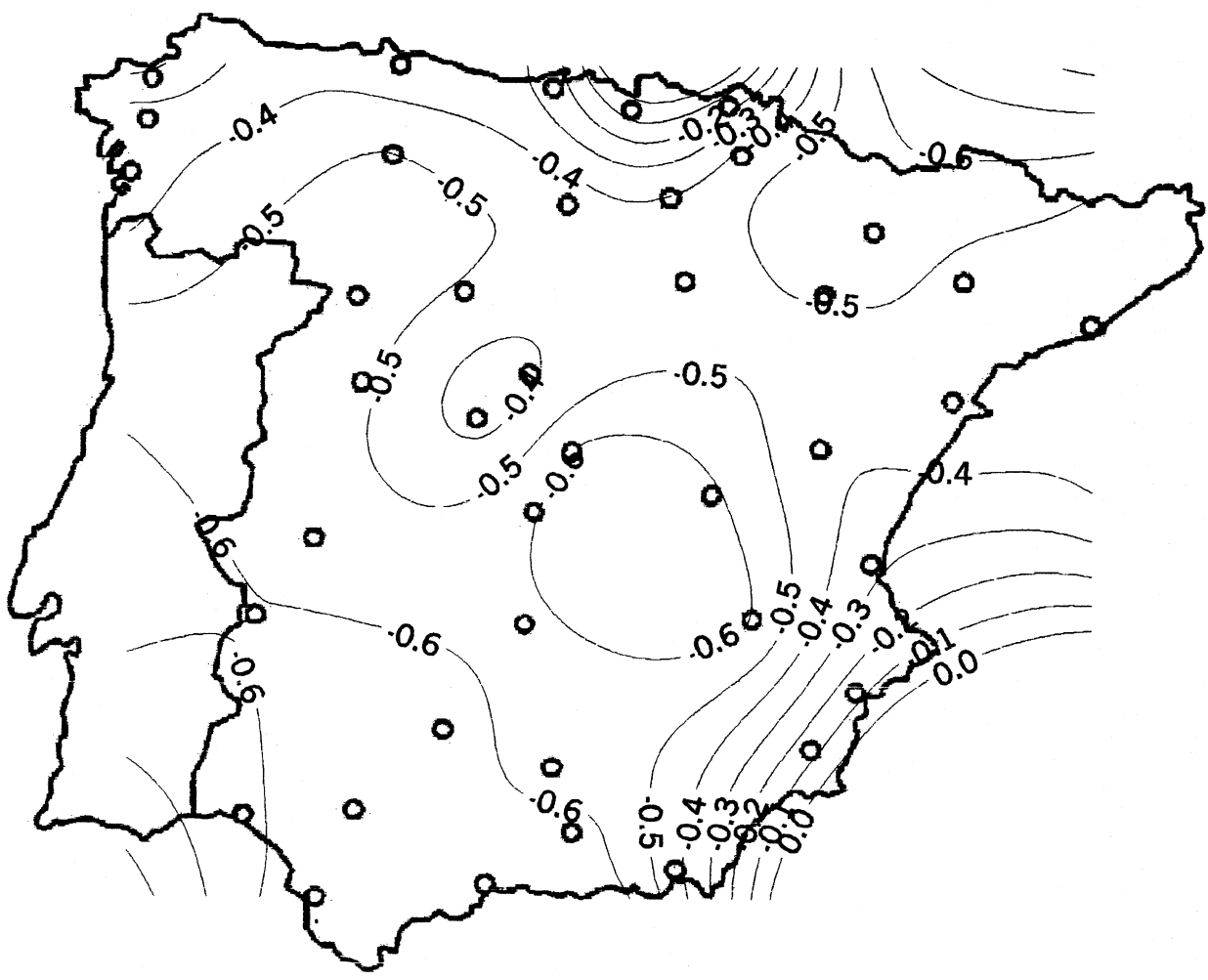

Figura 1. Valores de la $\mathrm{r}$ de Pearson de la correlación entre el índice NAO y la precipitación de enero (1900-1994).

En el mes de enero la Península Ibérica queda dividida en tres regiones, atendiendo a la correlación estudiada. Una estrecha franja septentrional presenta unos valores de $r$ comprendidos entre 0.0 y -0.3 , no significativos o sólo débilmente. Esto se debe a su situación en un área de transición en el dipolo NAO, entre la zona subtropical y la templada. La disposición de las isolíneas es zonal hasta el Pirineo navarro. En esta franja las mejores correlaciones corresponden al sector gallego, con valores en torno a -0.3 , como es el caso de Pontevedra o La Coruña, mientras que en la cornisa cantábrica los valores son menores, alrededor de -0.2 , como son los casos de Santander y Gijón. Por contra, las peores correlaciones se dan en Bilbao y San Sebastián, con -0.1.

La segunda región corresponde a la franja oriental de la Península Ibérica. Esta área presenta las isopletas en disposición submeridiana. La localización de este sector a sotavento de los flujos atlánticos provoca que sea poco sensible al dipolo NAO. Pero en enero el índice NAO presenta una cierta correlación en las ciudades de Barcelona (-0.4), Tortosa $(-0.5)$ y Almería (-0.4), mientras que las peores se encuentran en Alicante (-0.2) y Murcia $(-0.1)$. La ciudad de Valencia, con un valor de -0.3 , se halla, dentro de este sector oriental, a caballo entre una parte septentrional, donde la NAO es más influyente, y la mitad meridional, con correlaciones bajas o no significativas. La NAO no tendría influencia sobre 
gran parte del sureste peninsular, de Alicante a la costa oriental almeriense, totalmente a sotavento de la influencia atlántica, mientras que ya la ciudad de Almería sentiría su influjo vía Gibraltar.

La tercera región corresponde a la parte central y más meridional de la Península, especialmente su cuadrante suroeste. Es aquí donde se encuentran las mejores correlaciones, como pueden ser los casos de San Fernando, Sevilla, Toledo, Málaga y Madrid (-0.6). Toledo y gran parte de la Meseta meridional presentan valores entre -0.6 y -0.5 . Esta área es para el mes de enero la más sensible a la NAO, ya que la parte central y suroccidental de la Península queda expuesta a los flujos del oeste y suroeste procedentes del Atlántico, en especial del golfo de Cádiz, pluviométricamente activos en invierno.

\subsection{Febrero}

Las presiones medias en febrero en el período estudiado son de $1019.8 \mathrm{hPa}$ en Lisboa y de $1002.1 \mathrm{hPa}$ en Reykjavik, lo que supone una diferencia de más de $17 \mathrm{hPa}$. Los valores de la desviación típica para estos puntos de referencia son de $4.4 \mathrm{hPa}$ en Lisboa y de $10.2 \mathrm{hPa}$ en la capital islandesa. En el período estudiado el valor más alto del índice NAO correspondió a 1990, con 3.79, mientras que el valor menor se registró en 1947, con -4.78.

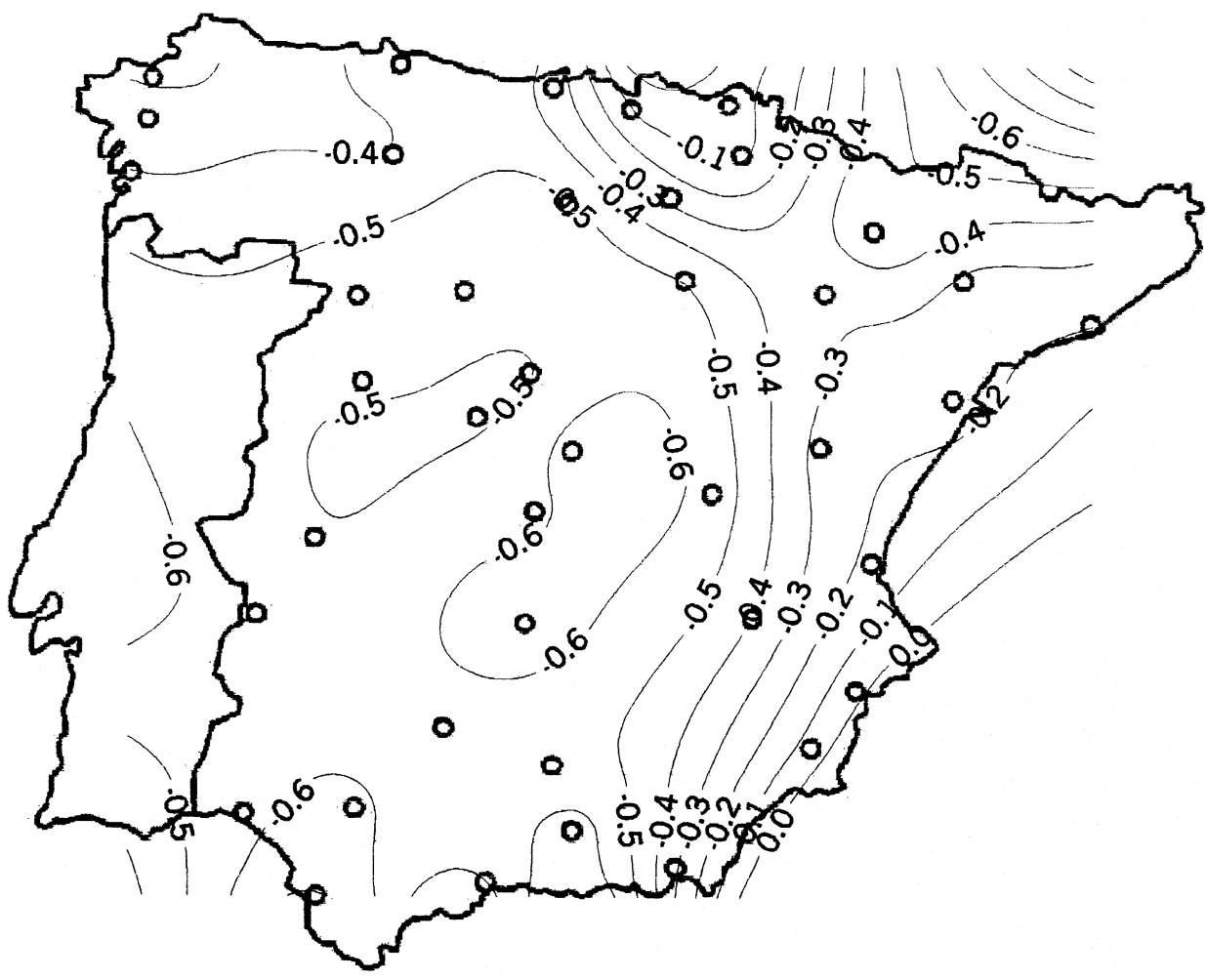

FIGURA 2. Valores de la $\mathrm{r}$ de Pearson de la correlación entre el índice NAO y la precipitación de febrero (1900-1994). 
En febrero la correlación entre el índice NAO y la precipitación da en todos los observatorios valores de $r$ de Pearson negativos, hallándose el valor más alto en Murcia (-0.05) y el más bajo en San Fernando (-0.71). Todos los valores son significativos, excepto en Alicante, Murcia, Valencia, San Sebastián, Bilbao y Pamplona, destacando, así, la zona más septentrional en su parte oriental y la franja oriental más meridional como los sectores sin vinculación a la NAO.

El mapa de isopletas de la Península muestra, al igual que en enero, tres áreas de influencia del índice NAO. La zona septentrional, con isopletas zonales y correlaciones bajas o no significativas, como las de Pamplona y Bilbao (-0.1). En esta región septentrional, en el sector occidental es donde se localizan las mejores correlaciones, como son los casos de Gijón (-0.4), Pontevedra y Santiago (-0.3). La franja oriental o mediterránea presenta una disposición meridiana de las isopletas, con unas correlaciones muy bajas o no significativas, incluso en Barcelona y Tortosa (-0.2), lo que constituye una diferencia con relación a enero. Las mejores correlaciones se dan en la Meseta, incluso la septentrional, y el suroeste, como en San Fernando (-0.7) o Madrid (-0.6).

\subsection{Marzo}

El comportamiento del índice NAO en el mes de marzo sigue unas pautas similares a las de los meses anteriores. Las correlaciones en marzo son todavía significativas en gran parte de la Península. La presión media en superficie en el observatorio de Lisboa para el período de estudio ha sido de $1017.3 \mathrm{hPa}$, con una desviación típica de $3.6 \mathrm{hPa}$. En Reykjavik la presión media ha sido de $1004.4 \mathrm{hPa}$, casi $14 \mathrm{hPa}$ menos que en la capital portuguesa, y ha presentado una desviación típica de $8.2 \mathrm{hPa}$. El valor más alto del índice NAO se dio en el año 1903, con 3.81, mientras que el valor más negativo o bajo se encuentra en 1916, con -4.63.

Los valores de la $r$ de Pearson entre la precipitación y el índice NAO son negativos en todos los observatorios estudiados. Pero no todos los valores son significativos estadísticamente, ya que en la parte más oriental de la Península los observatorios de Almería y Alicante (-0.14), Murcia (-0.07) y Tortosa (-0.13) quedan por debajo del umbral de significación, y tampoco son significativos en San Sebastián (-0.09), Pamplona (-0.15), Santander (-0.18) y Lérida (-0.21). Los observatorios con valores más significativos se sitúan en el suroeste de la Península, hasta - 0.7 en Sevilla, dándose igualmente valores buenos en el centro del país.

En la distribución de las isopletas sobre la Península se puede observar cómo los meses de febrero y marzo presentan comportamientos muy similares. Resulta interesante destacar que las correlaciones en estos meses son buenas en gran parte de la Meseta norte, en torno a -0.5 , como es el caso de Salamanca, Segovia o Zamora.

\subsection{Abril}

En el mes de abril Lisboa registra una presión media al nivel del mar de $1016.4 \mathrm{hPa}$, con una desviación típica de $2.1 \mathrm{hPa}$, mientras que en Reykjavik la presión media es de $1009.1 \mathrm{hPa}$, y su desviación típica de $5.2 \mathrm{hPa}$. El valor más alto del índice NAO corresponde al año 1947, con 5.04, mientras que el más bajo se registró en 1929 con -3.71.

En el mes de abril comienzan a aparecer correlaciones positivas, como es el caso de Lérida (0.03) y Bilbao (0.01). Por contra, las correlaciones mejores alcanzan valores de tan sólo -0.4, excepto en Sevilla, que presenta un valor de -0.5. De los 41 observatorios, 14 no 


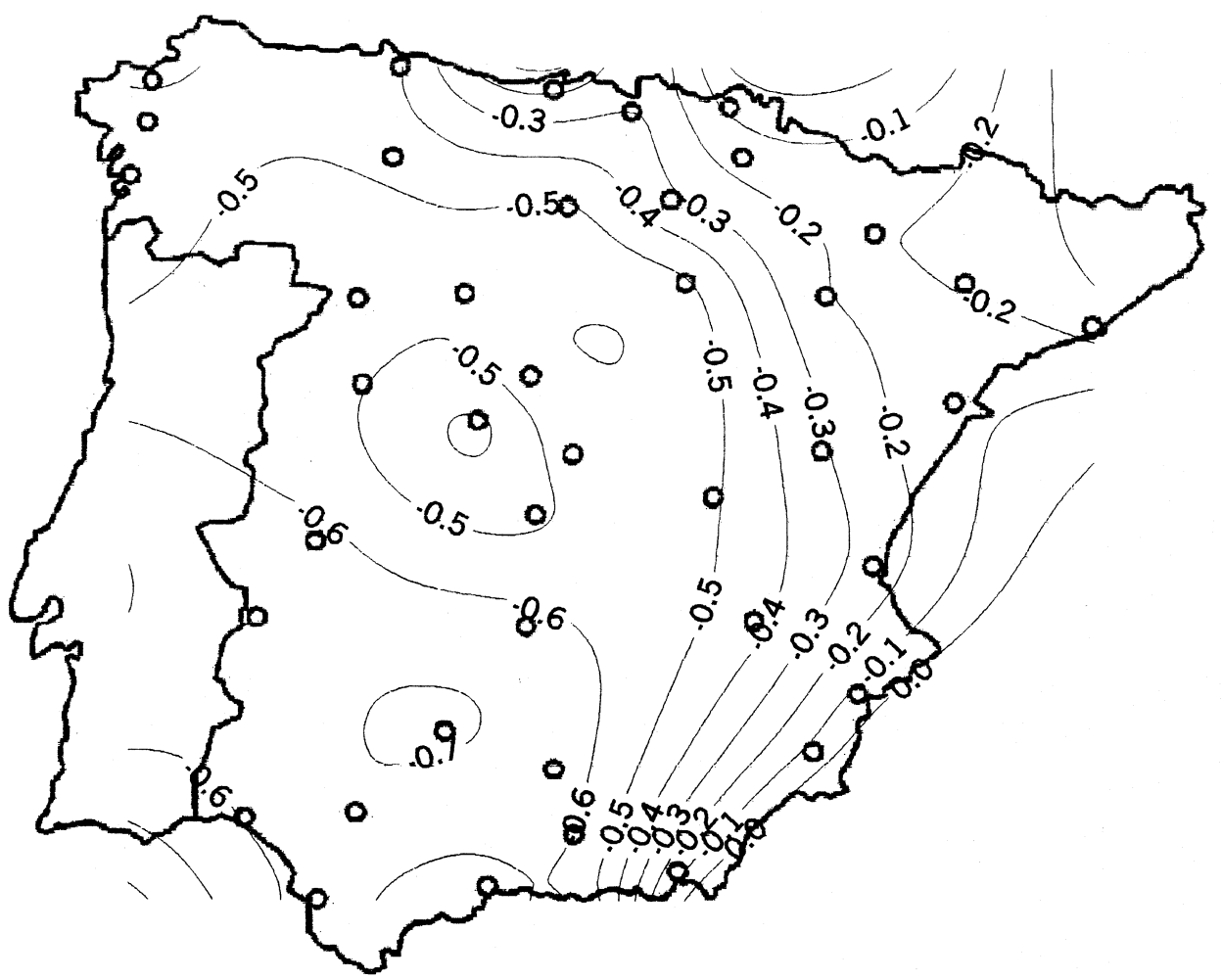

FiguRA 3. Valores de la r de Pearson de la correlación entre el índice NAO y la precipitación de marzo (1900-1994).

presentan significación estadística. Por áreas geográficas, los valores entre -0.2 y 0.2 , no significativos, se centran en la cornisa cantábrica y en el sector oriental de la Península. Algunas estaciones de estas áreas son San Sebastián, Bilbao, Tortosa y Murcia. Aparte de estas áreas, también puede comprobarse cómo estaciones que en los meses anteriores mostraban valores claramente significativos comienzan a tenerlos escasamente significativos. Por destacar algunas estaciones, pueden citarse León, Ávila y Lérida.

En consecuencia, las correlaciones son bajas en comparación con los meses invernales. Cabe decir que abril ya está dentro de la estación primaveral y, aunque la influencia de la NAO sigue siendo patente en la Península, resulta más débil que en meses anteriores. En la zona septentrional la dirección de las isopletas sigue siendo zonal. El sector oriental de la Península también continúa presentando isopletas meridianas, aunque los valores de la $r$ de Pearson quedan por debajo o en la frontera de la significación estadística. Por último, en el área central y suroccidental de la Península se siguen encontrando las mejores correlaciones, pero ya sólo con $r$ entre -0.4 y -0.3 . Este debilitamiento de la influencia de la NAO en la Península queda reflejado en la sucesión de situaciones advectivas de diferentes procedencias, especialmente meridianas, con consecuencias pluviométricas apreciables que se producen en abril. 


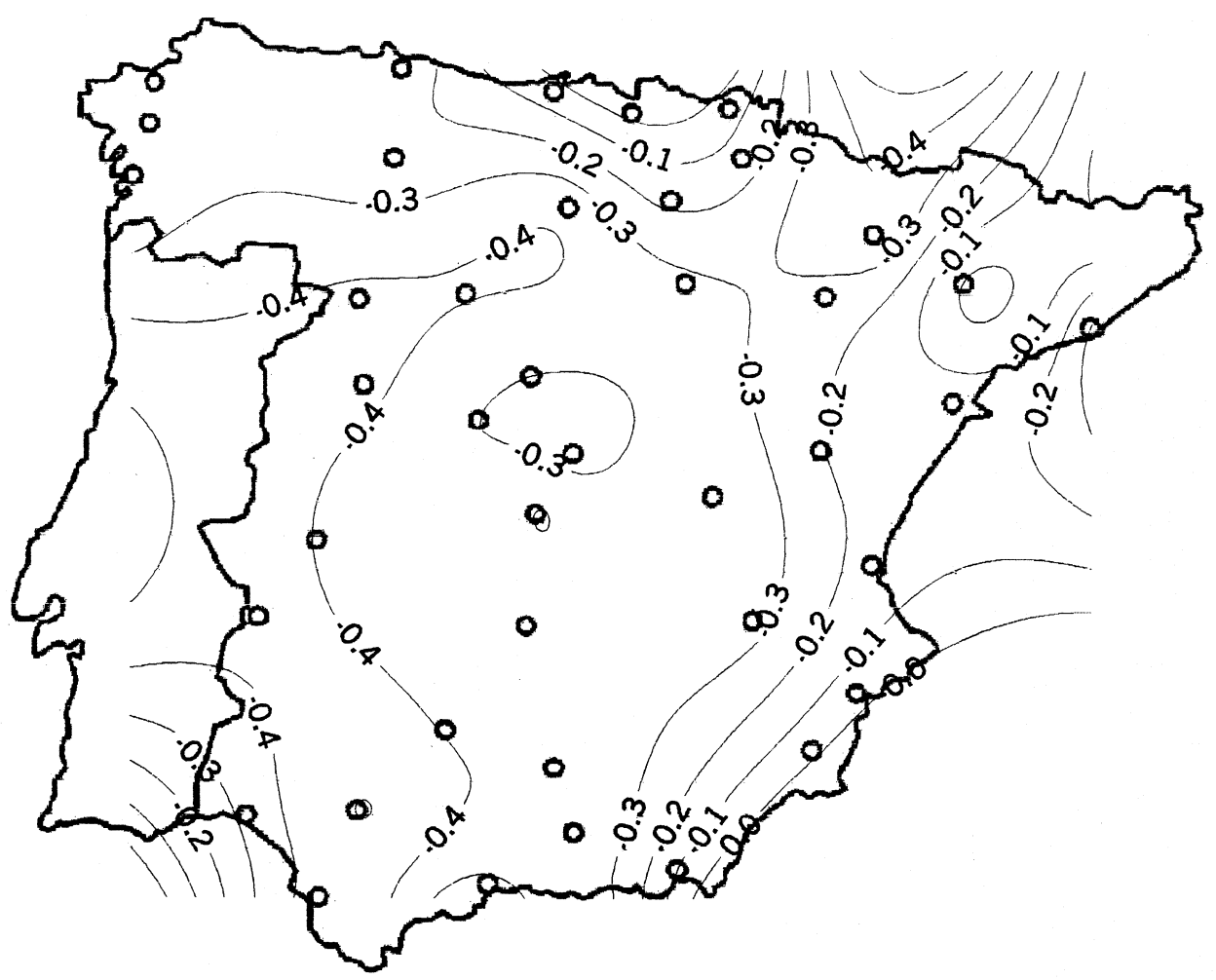

FIGURA 4. Valores de la $\mathrm{r}$ de Pearson de la correlación entre el índice NAO y la precipitación de abril (1900-1994).

\subsection{Mayo}

En el mes de mayo la presión media en Lisboa es de $1016.4 \mathrm{hPa}$, mientras que en Reykjavik es de $1013.0 \mathrm{hPa}$. La diferencia entre ambas ciudades se ha reducido drásticamente desde los meses de invierno, siendo la más baja de los doce meses del año. Las desviaciones típicas son de $1.6 \mathrm{hPa}$ para Lisboa y de $4.7 \mathrm{hPa}$ para Reykjavik. El valor más alto del índice NAO corresponde a 1956, con 4.60, mientras que el valor menor se dio en 1946, con -3.28 .

Para este mes, todos los valores de la $r$ de Pearson obtenidos para la correlación entre el índice NAO y la precipitación son negativos, excepto Zaragoza, que presenta un valor positivo (0.04), por lo que se convertirá en el más alto en este mes. El valor más bajo, es decir, la mejor correlación, corresponde a Cáceres, con -0.55. Las correlaciones son significativas en todos los observatorios, excepto en seis: Zaragoza (0.04), Teruel (-0.01), Santander (-0.19), Lérida (-0.12), Bilbao (-0.04) y Ávila (-0.15).

En algunos sectores la incidencia de la NAO se refuerza con relación a abril, tal como ocurre en la Meseta norte, donde Soria, Burgos, Segovia y Valladolid presentan valores comprendidos entre -0.40 y -0.50 . 


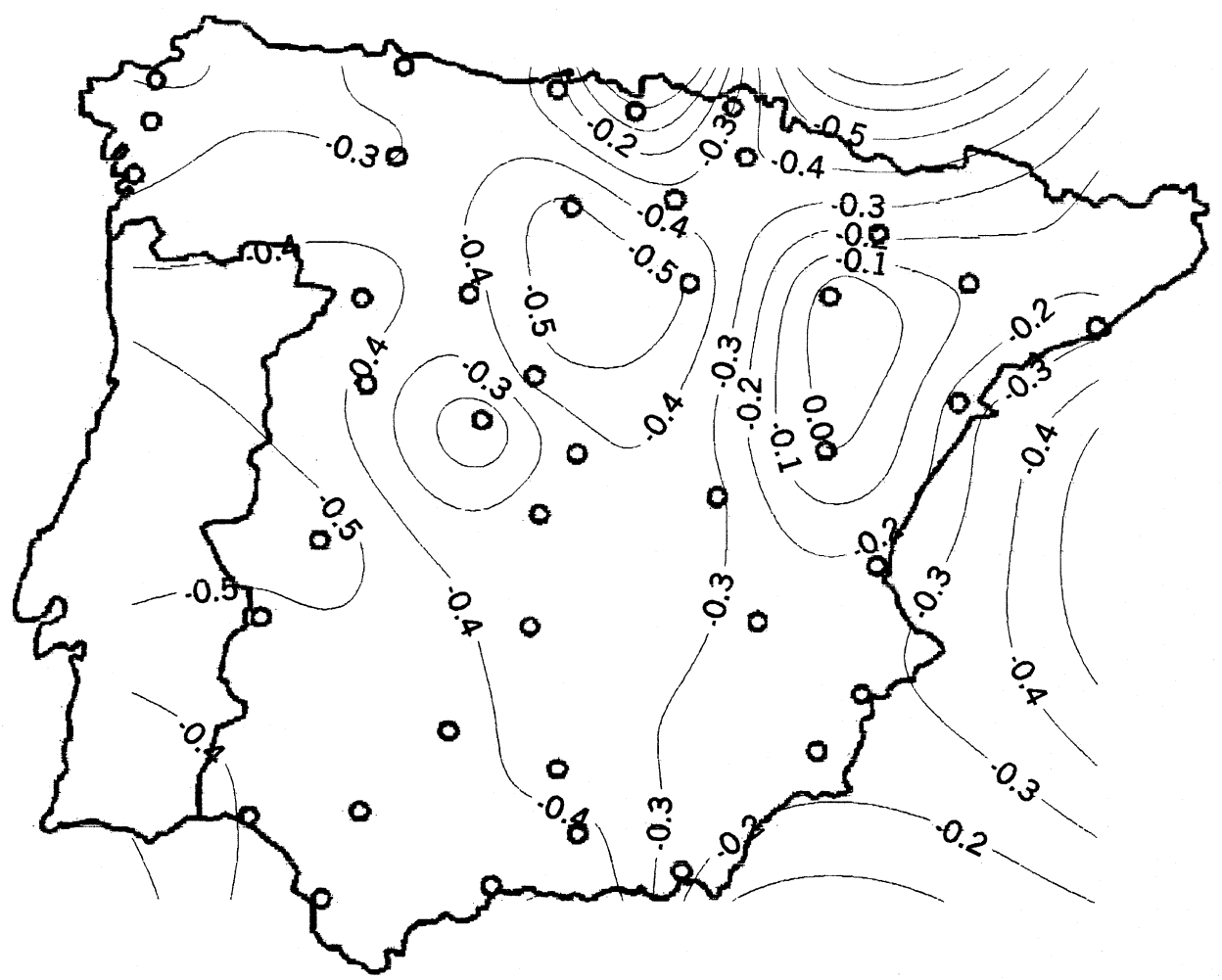

Figura 5. Valores de la $\mathrm{r}$ de Pearson de la correlación entre el índice NAO y la precipitación de mayo (1900-1994).

La zona norte de la Península sigue presentando unos valores de correlación bastante bajos, entre -0.1 y -0.3 . La parte mediterránea oriental continúa mostando una disposición isoplética meridiana. Llama la atención el núcleo aragonés con valores de $r$ prácticamente nulos, lo que supone una novedad respecto al mes de abril. Probablemente refleja el hecho del carácter local y convectivo de sus precipitaciones en mayo. El centro y suroeste peninsular muestra aún su vinculación con el dipolo NAO, aunque ya no acapara las mejores correlaciones.

\subsection{Junio, julio y agosto}

La práctica nula incidencia del patrón NAO en la explicación de las cantidades de precipitación del trimestre estival aconseja tratar conjuntamente sus tres meses. La propia naturaleza del dipolo, apenas definido en los meses de verano, y el peso de los procesos convectivos locales, así como la escasa pluviometría de la mitad sur peninsular, dan lugar a correlaciones no significativas en la mayoría de los observatorios. Sólo diez observatorios en junio, tres en julio y cuatro en agosto presentan valores de la $r$ de Pearson por encima del umbral de significación, aunque muy levemente. Los valores de $r$ son, de todos 


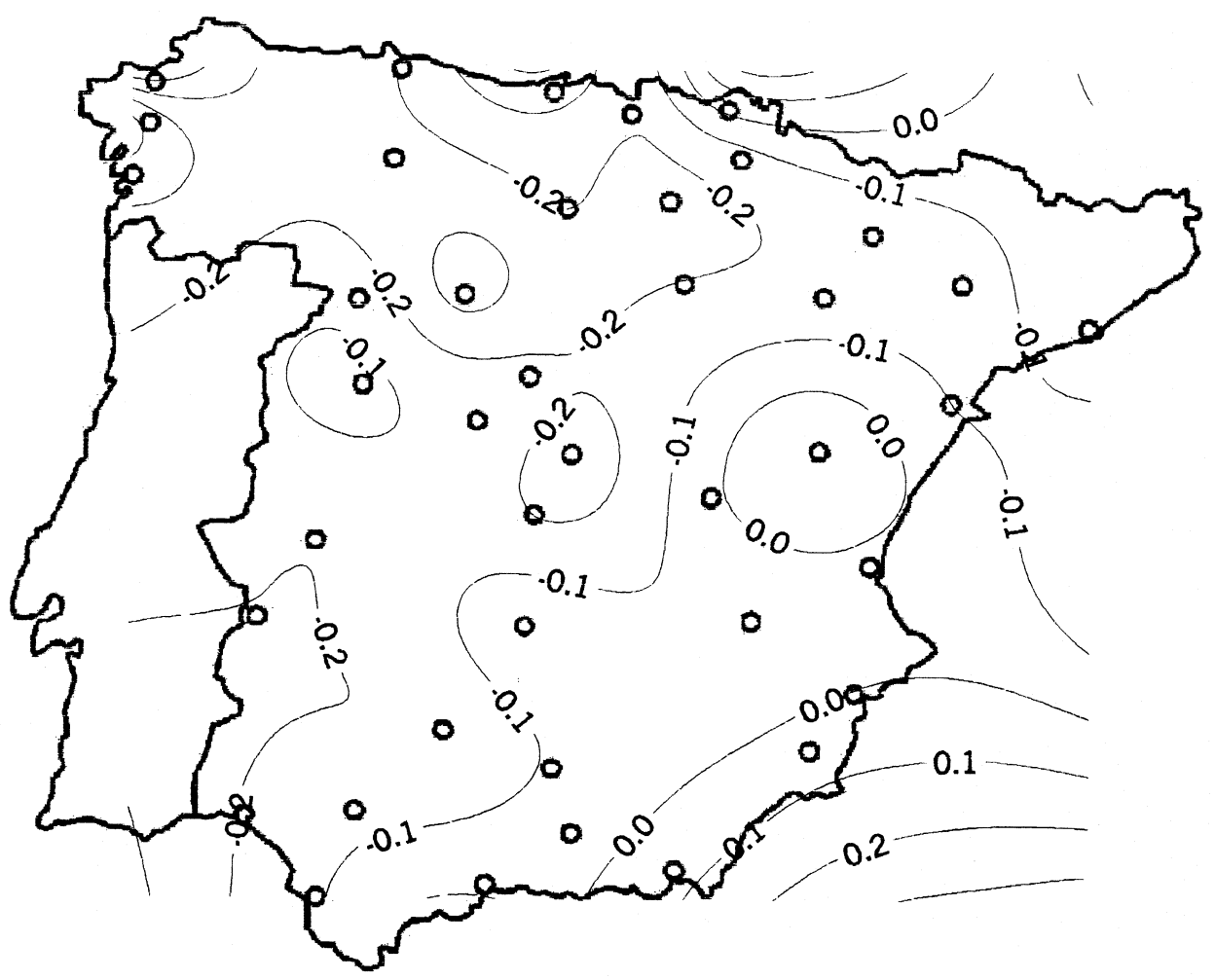

FIGURA 6. Valores de la $\mathrm{r}$ de Pearson de la correlación entre el índice NAO y la precipitación de junio (1900-1994).

modos, predominantemente negativos, con los más altos, en valor absoluto, en Pontevedra (-0.36), en junio, León (-0.31), en julio, y Málaga (-0.31), en agosto. La distribución de las isopletas no individualiza ninguna región, ni muestra patrones espaciales definidos en los citados meses.

Las presiones medias en junio son de $1017.5 \mathrm{hPa}$, en Lisboa, y $1011.0 \mathrm{hPa}$, en Reykjavik, con desviaciones típicas de $1.2 \mathrm{hPa}$ y $3.8 \mathrm{hPa}$, respectivamente. En julio, los correspondientes valores medios son de $1017.6 \mathrm{hPa}$, en Lisboa, y $1009.5 \mathrm{hPa}$, en Reykjavik, con desviaciones típicas de $0.9 \mathrm{hPa}$ y $3.5 \mathrm{hPa}$, respectivamente. En agosto, 1017.2 hPa, en Lisboa, y $1008.4 \mathrm{hPa}$, en Reykjavik, con desviaciones típicas de $0.8 \mathrm{hPa}$ y $3.4 \mathrm{hPa}$, respectivamente.

Los valores más elevados y más bajos del índice NAO en junio, para el período de estudio, correspondieron a 1961, con 3.41, y 1902, con-3.56. Los correspondientes a julio se produjeron en 1964 , con 2.78 , y 1968, con -3.33 . Y los de agosto, en 1919, con 3.56, y 1987, con -3.96 . 


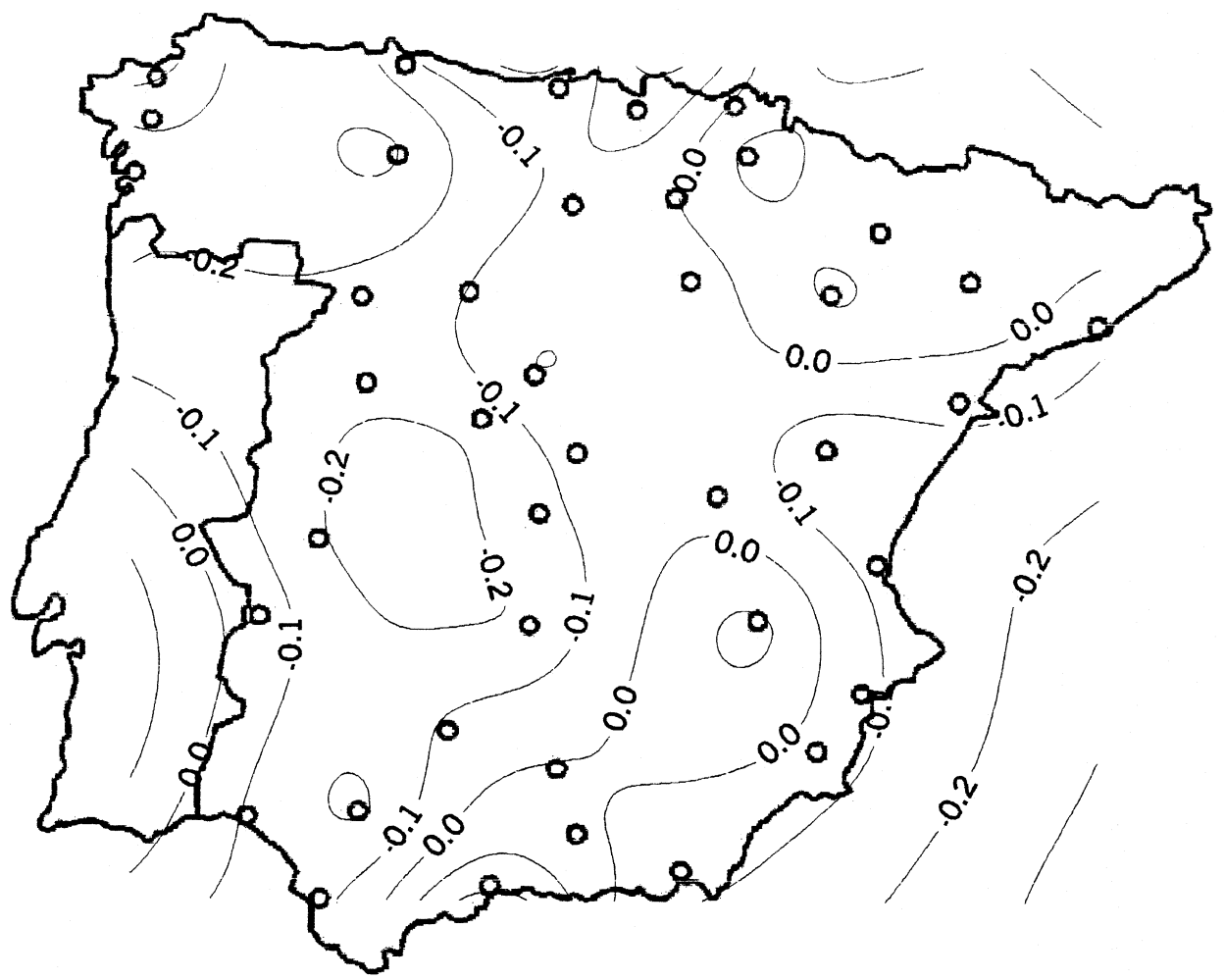

FIGURA 7. Valores de la r de Pearson de la correlación entre el índice NAO y la precipitación de julio (1900-1994).

\subsection{Septiembre}

Septiembre constituye un mes de transición entre el trimestre estival, sin señal NAO, y octubre, en que se inicia el período en el que la precipitación del centro y suroeste de la Península Ibérica tiene una dependencia importante del citado patrón de variabilidad de baja frecuencia.

La presión media registrada en Lisboa en este mes, durante el período de estudio, ha sido de $1017.5 \mathrm{hPa}$, mientras que la presión media en Reykjavik fue de $1005.9 \mathrm{hPa}$, lo que supone cerca de $12 \mathrm{hPa}$ de diferencia. La desviación típica de la presión atmosférica en Lisboa ha sido de $1.2 \mathrm{hPa}$ y de $4.5 \mathrm{hPa}$ en Reykjavik. El valor más alto del índice corresponde al año 1954, con 3.78, mientras que el más bajo se produjo en el año 1939, con -4.57 .

La $r$ de Pearson en el mes de setiembre es negativa excepto en dos puntos, San Sebastián (0.07) y Bilbao (0.08). La mejor correlación entre el índice NAO y la precipitación la registra Sevilla (-0.44). Los valores de la $r$ comienzan a ser débilmente significativos. Son 


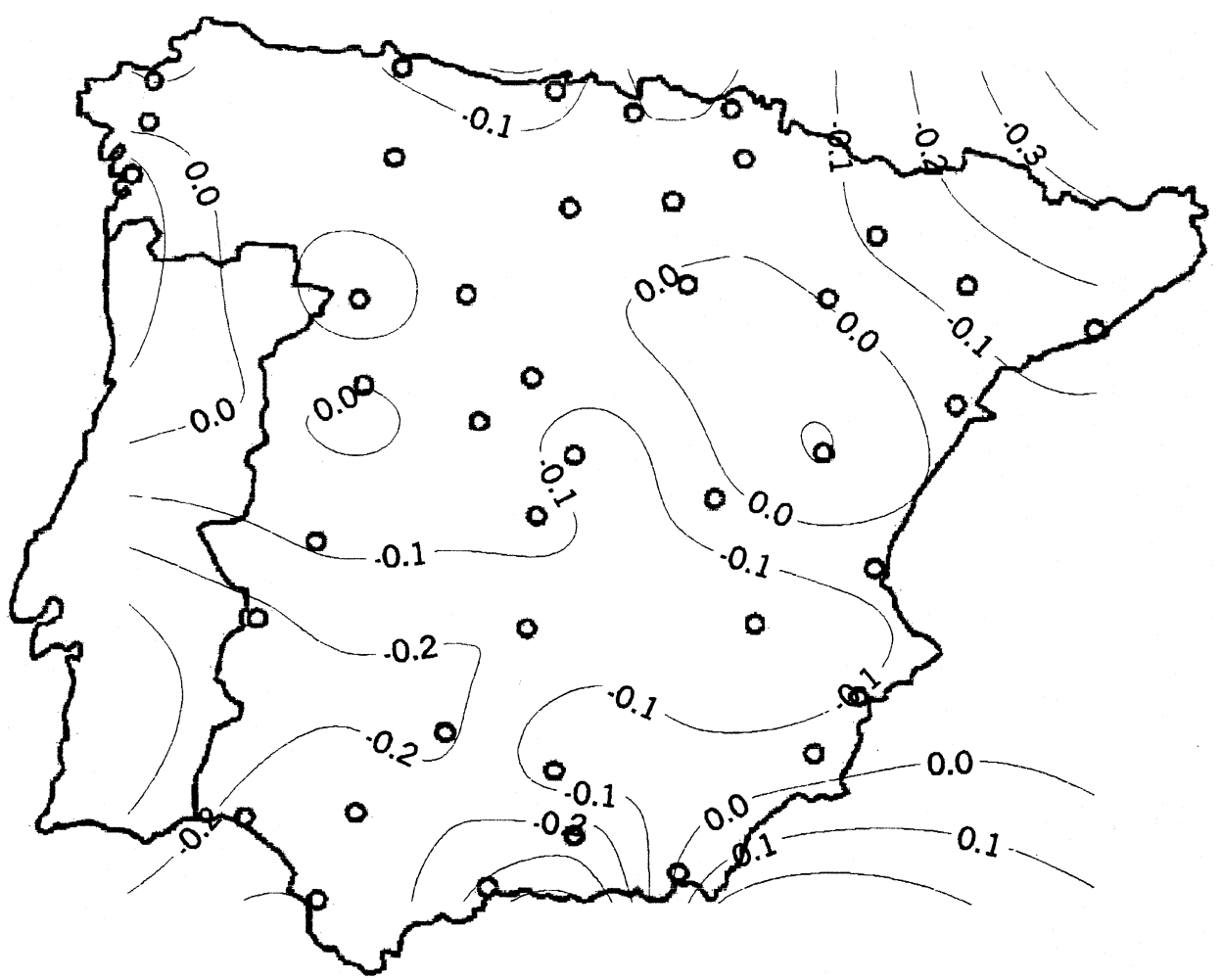

FIGURA 8. Valores de la r de Pearson de la correlación entre el índice NAO y la precipitación de agosto (1900-1994).

25 los observatorios que quedan por encima del umbral de significación. Del mismo modo, comienza a dibujarse un área central y suroccidental de la Península Ibérica que responde a la influencia de la NAO y una franja norte, especialmente el País Vasco, no correlacionada con el índice teleconectivo.

\subsection{Octubre}

Las presiones medias en Lisboa y Reykjavik en octubre son de $1017.5 \mathrm{hPa}$ y 1003.3 $\mathrm{hPa}$, con unas desviaciones típicas de $2.2 \mathrm{hPa}$ y $6.3 \mathrm{hPa}$, respectivamente. El valor más alto del índice NAO se dio en el año 1986, con 3.25, mientras que el más bajo corresponde al año 1922 , con un registro de -4.55 .

La mejor correlación entre el índice NAO y la precipitación se produce en San Fernando (-0.64), mientras que la peor corresponde al observatorio de Valencia (0.10). Todos los valores de la $r$ de Pearson son negativos, excepto en el este peninsular, donde Valencia y Murcia presentan valores positivos. Sólo siete observatorios no muestran valores significativos estadísticamente, los casos de Albacete, Barcelona, Bilbao, Murcia, San Sebastián, 


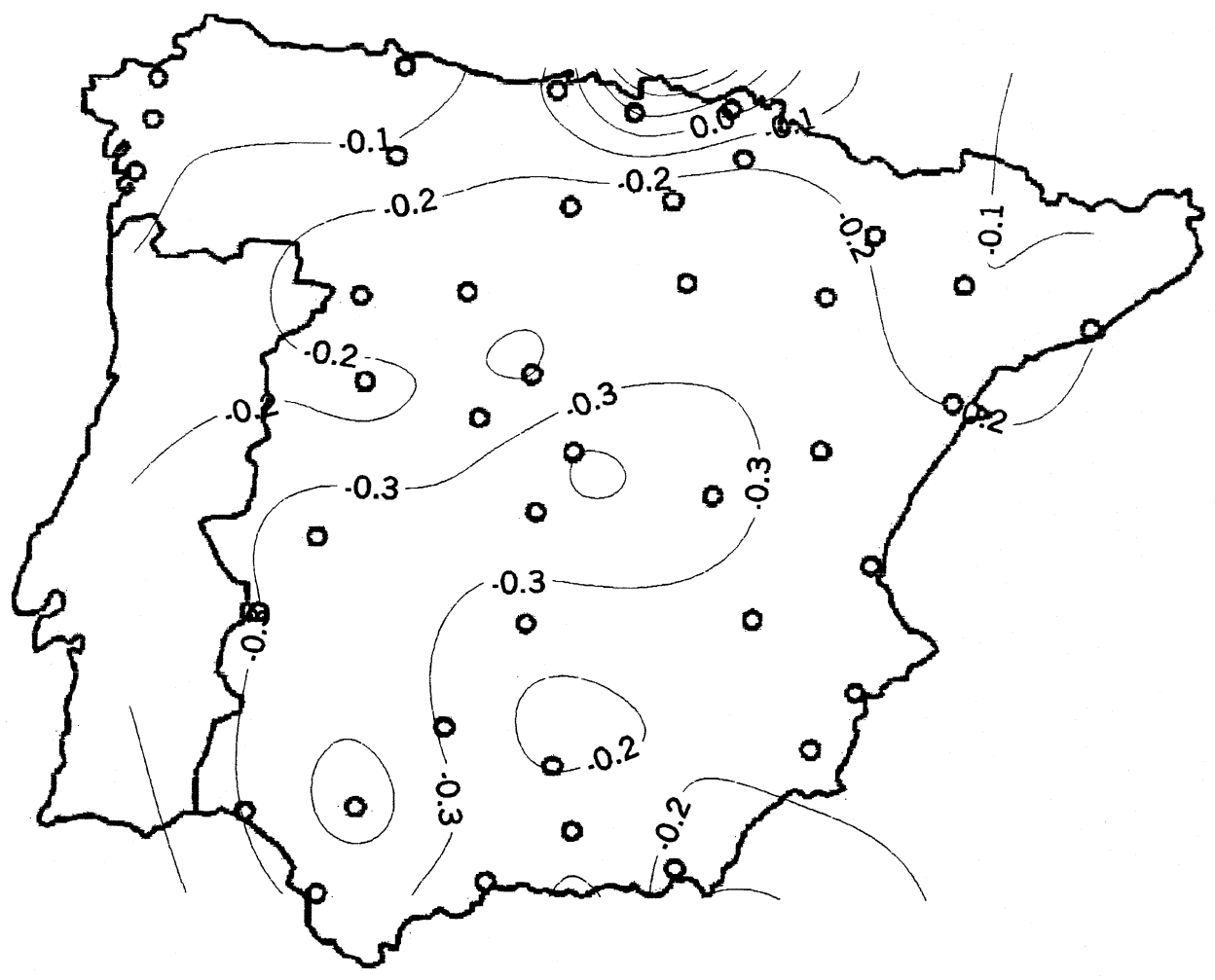

FIGURA 9. Valores de la $\mathrm{r}$ de Pearson de la correlación entre el índice NAO y la precipitación de septiembre (1900-1994).

Santander y Valencia, localizados en la cornisa cantábrica y en parte del sector oriental de la Península.

El mapa de isopletas de octubre muestra ya claramente la división de la Península en las tres regiones ya descritas en los primeros meses del año. La franja septentrional, donde las isopletas presentan una distribución zonal, no está correlacionada o sólo débilmente con la NAO. La franja oriental mediterránea, con isopletas submeridianas, tiene el mismo comportamiento que el sector cantábrico. Finalmente, en el centro y el suroeste peninsular se da ya una buena correlación negativa entre el índice NAO y la pluviometría.

\subsection{Noviembre}

En noviembre la presión media en Lisboa es de 1018.4 hPa, mientras que en Reykjavik es de $1002.4 \mathrm{hPa}$, es decir, $16 \mathrm{hPa}$ menos. Las desviaciones típicas son de $3.0 \mathrm{hPa}$ para Lisboa y de $6.8 \mathrm{hPa}$ para Reykjavik. El valor más alto del índice NAO corresponde a 1992, con 4.80, mientras que el menor se produjo en 1983, con -3.11 . 


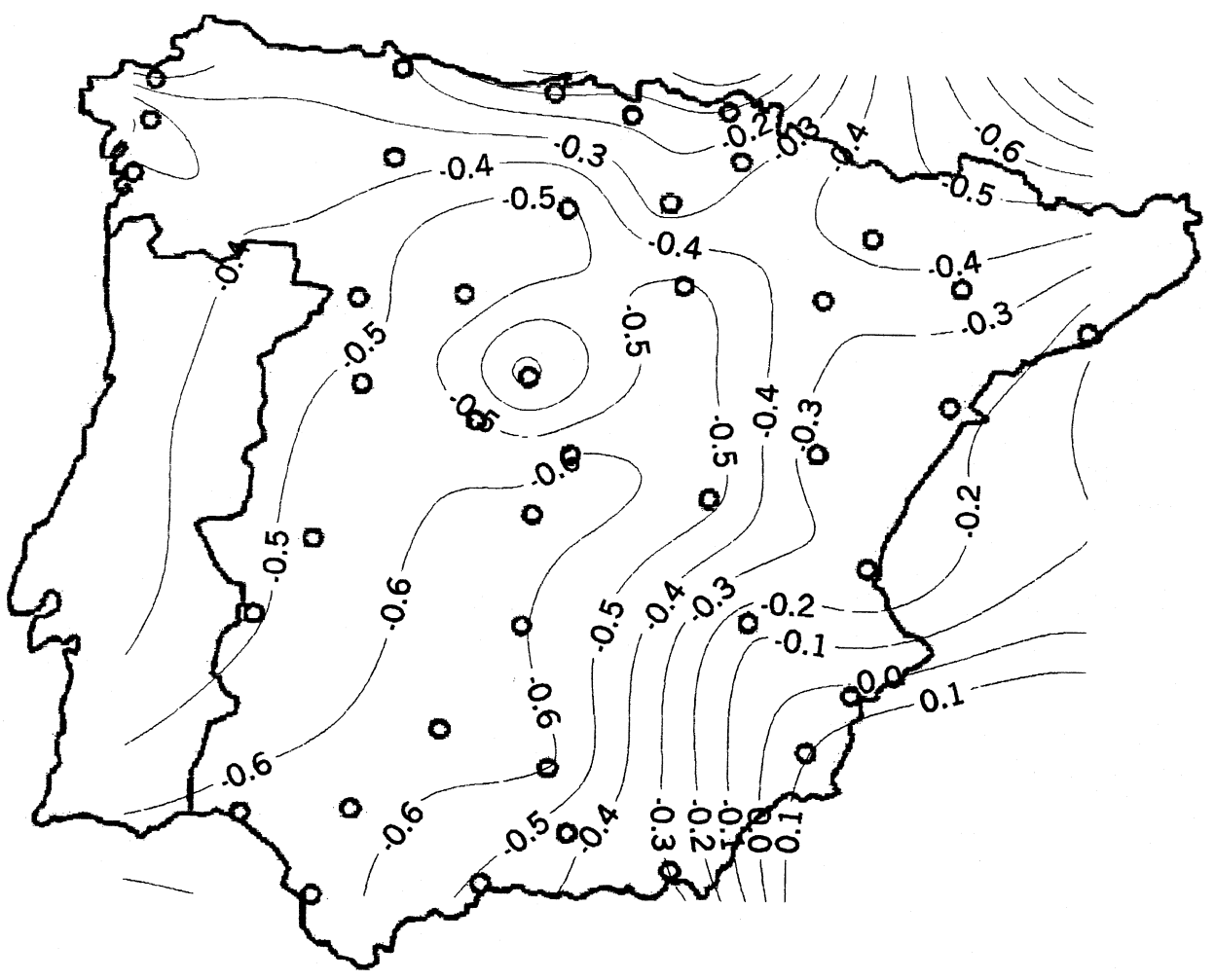

FIGURA 10. Valores de la r de Pearson de la correlación entre el índice NAO y la precipitación de octubre (1900-1994).

Todos los valores de la $r$ de Pearson son negativos, excepto dos, San Sebastián (0.09) y Bilbao (0.04). La mejor correlación entre el índice NAO y la precipitación en el mes de noviembre la registra Huelva (-0.61). Once observatorios no presentan valores estadísticamente significativos, los casos de Bilbao, La Coruña, León, Logroño, Murcia, Pamplona, Pontevedra, San Sebastián, Santander, Santiago y Valencia. Galicia y la franja cantábrica presentan valores no significativos en general, así como la región del sureste peninsular.

En conjunto, la bondad de las correlaciones disminuye ligeramente respecto a las correspondientes a octubre, excepto en el noreste peninsular. El cuadrante suroeste exhibe los valores de correlación más elevados.

\subsection{Diciembre}

En el mes de diciembre, y para el período de análisis, las presiones medias en Lisboa y en Reykjavik son de $1020.9 \mathrm{hPa}$ y $998.9 \mathrm{hPa}$, y sus desviaciones típicas, de $3.9 \mathrm{hPa}$ y 7.5 $\mathrm{hPa}$, respectivamente. La diferencia entre las medias supone nada menos que $22 \mathrm{hPa}$, la 


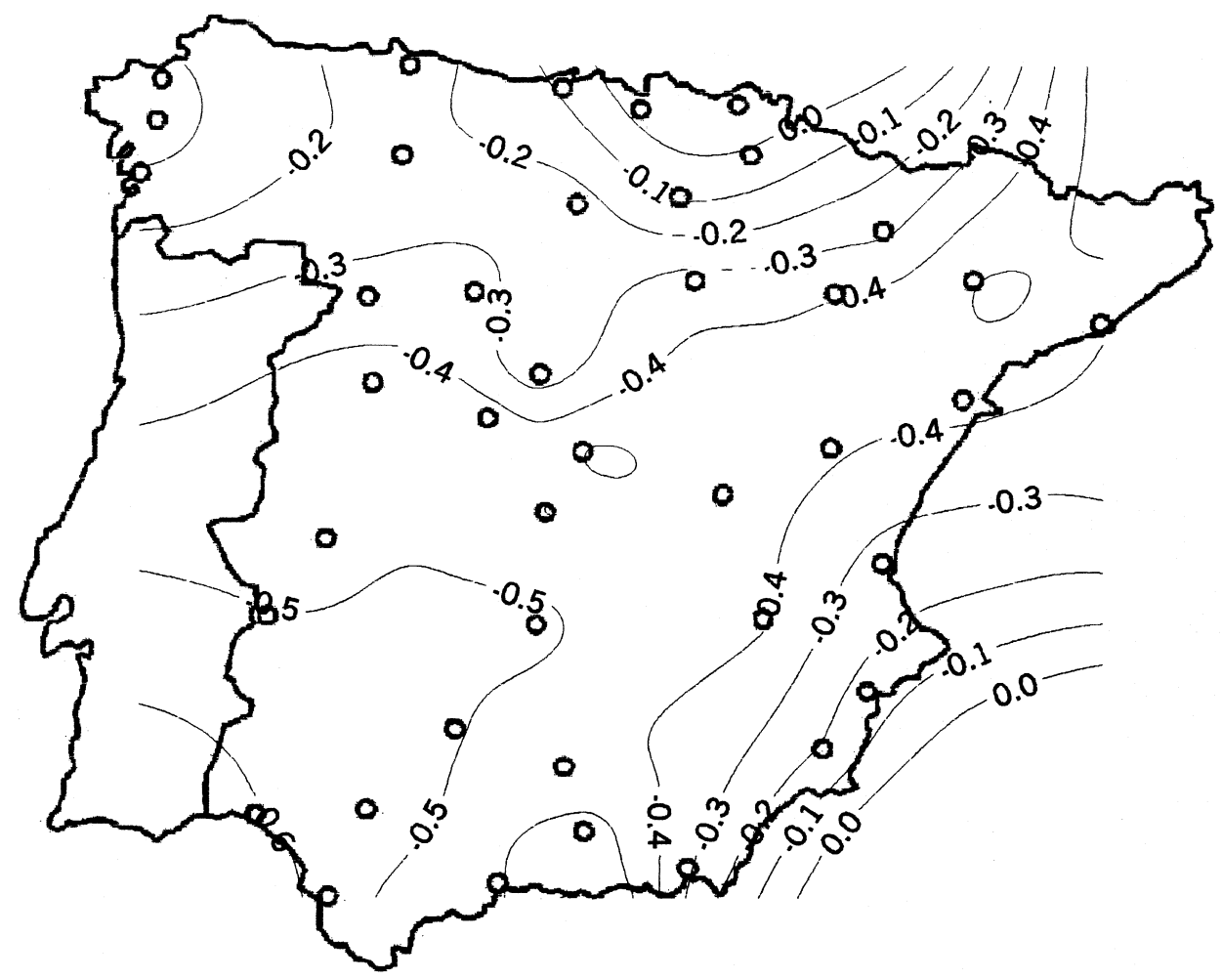

Figura 11. Valores de la $\mathrm{r}$ de Pearson de la correlación entre el índice NAO y la precipitación de noviembre (1900-1994).

segunda más elevada, después de la de enero. El valor más alto del índice NAO es 3.19, correspondiente a 1924, y el menor, -3.84, en 1976.

Todos los valores de la $r$ de Pearson obtenidos para la correlación entre el índice NAO y la precipitación en diciembre son negativos, excepto el correspondiente a San Sebastián (0.04). En la zona septentrional de España, donde los valores no alcanzan -0.3, la correlación no es estadísticamente significativa o sólo muy débilmente. Tampoco en gran parte de la franja oriental de la Península Ibérica, donde los valores de $r$ quedan por debajo de -0.4 , la correlación es apreciable. El centro y el cuadrante suroccidental peninsular presentan valores de $r$ entre -0.5 y -0.7 , claramente significativos. Las isopletas del coeficiente de correlación dibujan un haz zonal con elevado gradiente en el norte de España, separando la franja septentrional de clima marítimo del resto del país, de clima mediterráneo. Hacia el este, el citado haz se curva en dirección sur para individualizar las tierras orientales próximas al mar Mediterráneo. Los valores de $r$ más significativos ocupan las regiones de la Meseta, excepto su parte más septentrional, puntos del valle del Ebro y Andalucía occidental. Ciudad Real (-0.69), Sevilla (-0.68) y Madrid (-0.67) ofrecen las mejores correlaciones (Martín Vide et al., 1999). 


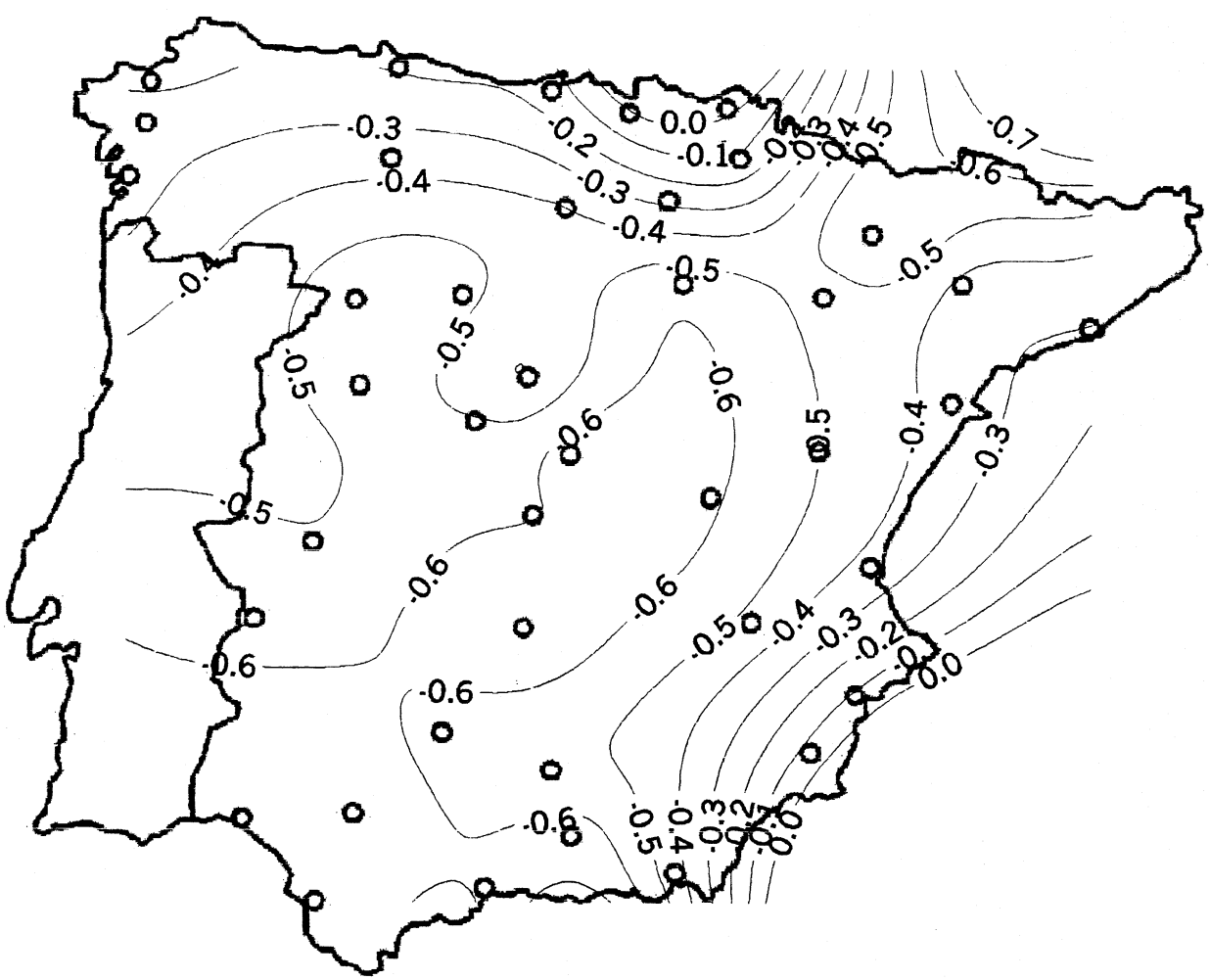

FIGURA 12. Valores de la r de Pearson de la correlación entre el índice NAO y la precipitación de diciembre (1900-1994).

En resumen, el territorio español peninsular queda dividido en las tres áreas ya apreciadas en los meses de la mitad fría del año. La mejor correlacionada y más extensa es el centro y suroeste, salvo alguna excepción, como en el valle del Ebro, en la que los temporales atlánticos del oeste y suroeste producen la mayor parte del total pluviométrico. Esto suele ocurrir con depresiones localizadas hacia el oeste y suroeste de la Península Ibérica, asociadas, pues, a índices NAO negativos. Parece incluso insinuarse en el trazado de las isopletas un eje diagonal suroeste - noreste, coincidente con la dirección de los flujos húmedos procedentes del suroeste.

\section{Conclusiones}

La Oscilación del Atlántico Norte, como patrón de variabilidad de baja frecuencia, explica una parte sustancial de la variabilidad pluviométrica del centro y suroeste de la España peninsular en los meses de octubre a marzo, inclusives. La correlación entre el índice NAO y la precipitación en los citados meses es negativa y claramente significativa en la región citada, con valores generalmente comprendidos entre -0.5 y -0.7 . Los tempo- 
rales del suroeste, que aportan un alto porcentaje de las lluvias del sur y el centro peninsular, vinculados a depresiones localizadas hacia el área del golfo de Cádiz o sus proximidades, explican la mencionada dependencia. En el resto de la España peninsular, tanto la franja septentrional como el área oriental, con isopletas de la $r$ de Pearson zonales, en el primer caso, y submeridianas, en el segundo, muestran una dependencia débil o no significativa con respecto al patrón NAO. En abril y mayo comienza a debilitarse la correlación entre el índice NAO y la precipitación en toda España. En el trimestre estival, junio, julio y agosto, no existe señal NAO en ninguna región, mientras que el mes de septiembre puede considerarse de transición entre el verano y el período de clara influencia de la NAO.

\section{Agradecimientos}

La presente investigación se ha realizado en el marco del proyecto CLI98-0930-C02-02 (CICYT), del Grup de Climatologia (Generalitat de Catalunya) y del Parc Científic (Universidad de Barcelona). Los autores desean, asimismo, agradecer al profesor Jorge Olcina su ayuda técnica.

\section{Bibliografía}

CAPEL MOLINA, J.J. (2000): El clima de España y Portugal, Barcelona, Ariel.

GIL OLCINA, A. y MORALES GIL, A. (1989)(eds.): Avenidas fluviales e inundaciones en la cuenca del Mediterráneo, Universidad de Alicante y CAM.

HURRELL, J.W. (1995): «Decadal trends in the North Atlantic Oscillation: regional temperature and precipitation». Science, 269, 676-679.

KATZ, R.W. (1988): «Use of cross-correlations in the search for teleconnections». Journal of Climatology, 8, 241-253.

LAITA, M. (1995): El fenómeno del Niño y su influencia climática en el Mediterráneo occidental, Palma, Universidad de las Islas Baleares (Tesis doctoral inédita).

MARTÍN VIDE, J. (1996): «Decálogo de la pluviometría española», en Marzol et al. (eds.), Clima y agua. La gestión de un recurso climático, 15-24, Universidad de La Laguna, AGE.

MARTÍN VIDE, J. y GÓMEZ, L. (1999): «Regionalization of peninsular Spain based on the length of dry spells». International Journal of Climatology, 19, 537-555.

MARTÍN VIDE, J.; BARRIENDOS, M.; PEÑA, J.C.; RASO, J.M.; LLASAT, M ${ }^{\text {a }}$ C. y

RODRÍGUEZ, R. (1999): «Potencialidad del índice NAO en la previsión de episodios de alta pluviometría en España». Gerencia de riesgos, 67, 19-29, Madrid, Fundación Mapfre Estudios.

MINISTERIO DE MEDIO AMBIENTE (1996): Homogeneidad y variabilidad de los registros históricos de precipitación de España, Madrid.

MEEHL, G.A. y VAN LOON, H. (1979): «The Seesaw in Winter Temperatures between Greenland and Northern Europe. Part III: Teleconnections with lower latitudes». Monthly Weather Review, 107, 1095-1106.

PARKER, D.E. y FOLLAND, C.K. (1988): «The Nature of Climatic Variability». Meteorological Magazine, 117, 201-210.

RODÓ, X. et al. (1997): «Variations in seasonal rainfall in Southern Europe during the present century relationships with the North Atlantic Oscillation and the El Niño-Southern Oscillation». Climate Dynamics, 13, 275-284. 
VAN LOON, H. y ROGERS, J.C. (1978): «The Seesaw in Winter Temperatures between Greenland and Northern Europe. Part I: General Description». Monthly Weather Review, 106, 296-310.

WMO (1996): The Global Climate System Review. Climate System Monitoring, June 1991November 1993, 819.

ZORITA, E. et al. (1992): «The atmospheric circulation and sea surface temperature in the North Atlantic area in winter: their interaction and relevance for Iberian precipitation». Journal of Climate, 5, 1097-1108. 